\title{
Estimation of Collision Frequency in the Upper $D$ and $E$ Regions from LF Wave by Means of a Rocket Experiment
}

\author{
Isamu NagANo, * Masayoshi Mambo, ${ }^{*}$ and Iwane KImURA** \\ *Department of Electrical Engineering, Kanazawa University, Kanazawa, Japan \\ **Department of Electrical Engineering, Kyoto University, Kyoto, Japan
}

(Received October 2, 1979)

\begin{abstract}
A method of estimation of the collision frequency in the lower ionosphere using the field intensity of a ground based LF signal observed by a rocket is described. The magnetic intensity profiles, in the ionosphere, of a ground based signal observed by a rocket and the electron density simultaneously observed at several altitudes are analyzed by iterative calculations based on a simple WKB solution. The collision frequency for mono-energetic electrons, $v_{\mathrm{m}}$, in the upper $D$ and $E$ regions was actually estimated from the altitude profile of the right handed circularly polarized component of a $40 \mathrm{kHz}$ ground based signal observed by means of the K-9M-53 rocket. As a result, the following relationships were obtained; $v_{\mathrm{m}}=4.8 \times 10^{5} \mathrm{P}$ for the upper $D$ region and $v_{\mathrm{m}}=11.0 \times 10^{5} \mathrm{P}$ for the $E$ region, where P (Newton $\left./ \mathrm{m}^{2}\right)$ was taken from CIRA (1972).
\end{abstract}

\section{Introduction}

In investigating the collision damping of a radio wave in the ionosphere, the collision frequency $v_{\mathrm{m}}$ for mono-energetic electrons has been often used, which is closely proportional to the atmospheric pressure, $\mathrm{P}\left(\mathrm{Newton} / \mathrm{m}^{2}\right)$; e.g., $v_{\mathrm{m}}=C \times 10^{5} \mathrm{P}$, where the value of $C$ is called the collision proportionality factor. The value of $C$ has been determined from laboratory measurements of the cross sections of nitrogen and oxygen (Benson, 1964; Thrane and Piggott, 1966). Recently, Mechtly (1974) obtained the value of $C$ from simultaneous rocket measurements of Faraday rotation and differential absorption of a HF ground based signal. According to him, $C$ varies from 3.0 to 9.0 as a function of altitude. On the other hand, there were several definite values of $C$ used for the analyses of collisional absorption of radio wave in the lower ionosphere, such as 6.38 (Thomas and Harrison, 1970), 8.0 (Beynon and Williams, 1976) and 7.5 (Bremer and SINGER, 1977).

In this paper, a method is described for estimating the collision frequency $v_{\mathrm{m}}$ from the field intensities of a whistler mode signal observed in the ionosphere by means of a simple rocket borne instrument and a result obtained by the K-9M-53 rocket experiment is presented. In our method of obtaining the collision frequency, it is required that the electron densities at several independent altitudes are known, i.e., are measured. Analyses of these signal intensities are based on a simple WKB solution. In general it is well-known 
that a generalized WKB solution for oblique incidence of VLF wave in the lower ionosphere is too complicated to apply to practical analyses. However, we can derive a simple formula which is valid only for a whistler mode in a medium where the refractive index is much greater than unity (Appendix A). Therefore an altitude range for which the collision frequency is estimated by this formula depends on the signal frequency to be used and on the electron density profile. In our experiment, the lower limit in altitude was $90 \mathrm{~km}$.

The measurement using the K-9M-53 rocket was made of the polarization of a $40 \mathrm{kHz}$ signal transmitted from Kemigawa near Tokyo on August 26, 1975. From this experiment the electron density profile in the $D$ region was measured and was already reported in another paper by the present authors (NAGANO et al., 1978), which will be referred to as paper 1. Hereafter, the collision frequency deduced from the same polarization data above the altitude $90 \mathrm{~km}$ will be presented.

\section{Experimental Data}

To estimated the collision frequency, we have a measured altitude profile of the $40 \mathrm{kHz}$ signal intensities with right-handed circular polarization in the ionosphere and electron densities at three altitudes.

\subsection{Intensities of the LF signals}

The K-9M-53 rocket was launched with an initial elevation angle of $80^{\circ}$ toward an azimuthal direction of $145^{\circ}$ clockwise from the north at 19:40 (J.S.T.) on August 26, 1975 from Kagoshima Space Center $\left(131^{\circ} 04^{\prime} \mathrm{E}, 31^{\circ} 15^{\prime} \mathrm{N}\right)$. It reached the maximum altitude of $350.8 \mathrm{~km}$ and fell into the sea $350 \mathrm{~km}$ from the launching site. The trajectory of the flight and a ray path of the received signal from the transmitter are shown in Fig. 1.

The signal $(40 \mathrm{kHz}, \mathrm{JG} 2 \mathrm{AS})$, which was transmitted from Kemigawa near Tokyo,

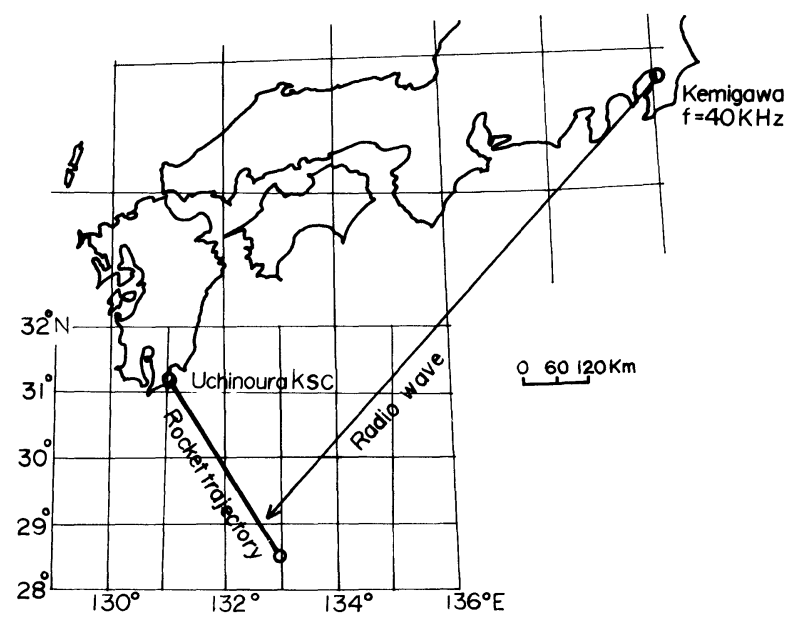

Fig. 1. Trajectory of the K-9M-53 rocket and a ray path of the received signal from the transmitter. 


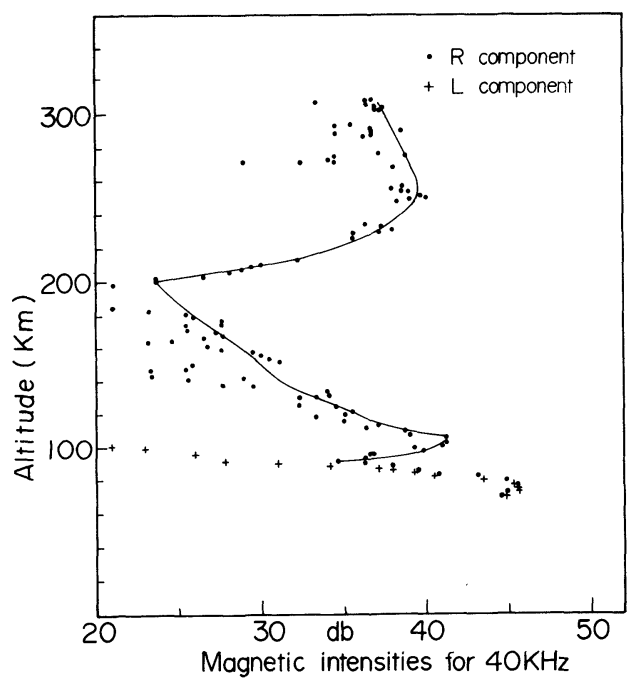

Fig. 2. Magnetic field intensities of the right- and left-handed circularly polarized components of the $40 \mathrm{kHz}$ signal observed by the K-9M-53 rocket during the descent flight $(0 \mathrm{~dB}=1 \mu \mathrm{V} / \mathrm{m})$.

Table 1. Electron densities used in this analysis.

\begin{tabular}{lcll}
\hline & $\begin{array}{c}\text { Altitude } \\
(\mathrm{km})\end{array}$ & $\begin{array}{c}\text { Electron density } \\
(\mathrm{els} / \mathrm{cc})\end{array}$ & \multicolumn{1}{c}{ Method for measurement } \\
\hline (a) & 90 & $1.0 \times 10^{3}$ & VLF polarization and absorption by K-9M-53 \\
(b) & 106 & $1.2 \times 10^{5}$ & Ionogram at Yamagawa \\
(c) & 260 & $5.0 \times 10^{5}$ & Gyro plasma probe by K-9M-53 \\
\hline
\end{tabular}

was successfully observed during the descent flight from the apex down to $70 \mathrm{~km}$. The LF signal could be separated into right- and left-handed circularly polarized components by crossed loop antennas on board the rocket (NAGANO et al., 1976). Figure 2 shows altitude plots of the wave magnetic field components in a plane perpendicular to the rocket axis during the descent flight. Since the precession angle of the rocket was less than $15^{\circ}$ and was stable, the values of measurement can be regard as the horizontal components. As is seen in Fig. 2, the signal was observed in the right-handed circularly polarized mode, namely in the whistler mode above an altitude of $90 \mathrm{~km}$ where our method is valid.

\subsection{Electron densities}

The electron densities at three altitudes are presented in Table 1. The electron density (a) in Table 1 was obtained by analyzing the polarization and absorption data below the altitude $90 \mathrm{~km}$ in Fig. 2 with a full wave technique (paper 1). The location of the K-9M-53 rocket in descent flight was far from the launching site, but the latitude dependence of the $f_{x} \boldsymbol{E}$ was very small as shown in Fig. 3 a, hence we estimated the electron density (b) from the ionogram which was obtained at Yamagawa observatory near the launching site (Fig. $3 b)$. (c) was measured by a gyro plasma probe on board the same K-9M-53 rocket. 


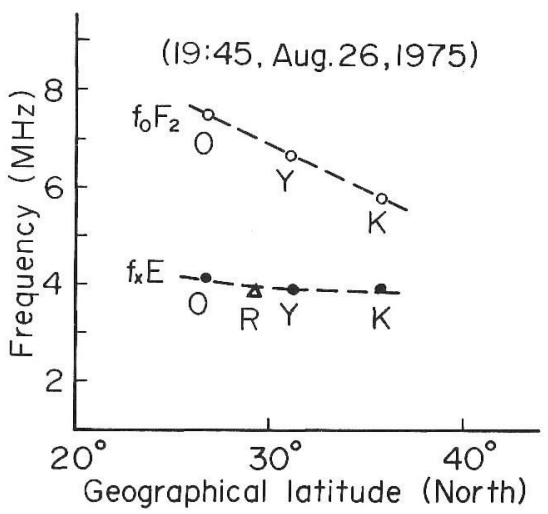

Fig. 3a. Geographic latitude variations of $f_{x} E$ and $f_{0} F_{2}$. (O), Okinawa; (Y), Yamagawa; (R), Descent site; (K), Kokubunji.
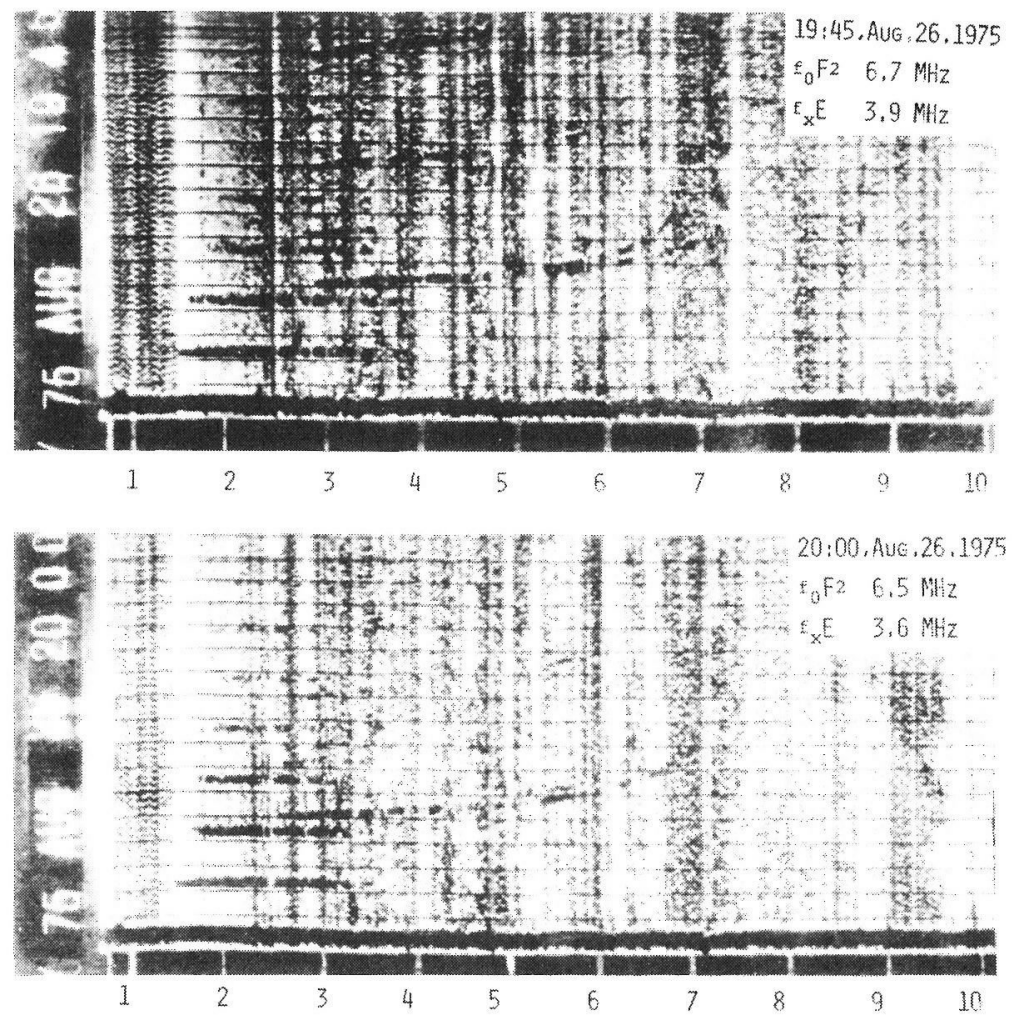

Fig. 3b. Ionograms at Yamagawa Radio Observatory near the launching site. 


\section{Method of Derivation of the Collision Frequency}

3.1 Intensity of the wave field in the whistler mode estimated by the WKB approximation

In the lower ionosphere below the altitude about $90 \mathrm{~km}$, the wave field in the VLF range is generally composed of the four characteristic waves, which are slightly coupled with each other when a variation of the medium per wave length is not so small and/or when the polarizations become nearly equal. Calculation of the fields in such a complex region should be made by a full wave technique. However, in the case where a single characteristic wave propagates as a dominant mode, and in the case where the values of $X$ $\left(\equiv \omega_{\mathbf{p}}{ }^{2} / \omega^{2}\right)$ and $Z\left(\equiv v_{\text {eff }} / \omega\right)$ in the ionosphere vary slowly enough with respect to altitude, WKB solutions are derived by BuDDEN (1961), equation (18-86), where $X, Z, \omega_{\mathrm{p}}$ and $v_{\text {eff }}$ are the commonly used notations, and will be defined later.

In our problem, as is seen in Fig. 2, the whistler mode with a right-handed circular polarization is a dominant wave above the altitude $90 \mathrm{~km}$, where the absolute value of $1-j Z$ is sufficiently less than $X$ and $Y\left(\equiv \omega_{\mathrm{H}} / \omega\right)$ because the electron density is greater than $10^{3} / \mathrm{cc}$ and the wave angular frequency $\omega$ is less than the gyro angular frequency $\omega_{\mathbf{H}}$. Then, we can give a more simplified formula for an upgoing whistler mode by using such parameter conditions (Appendix A), i.e., the horizontal magnetic component is given by

$$
H \propto q^{1 / 2} \exp \left[-j k_{0} \int q \mathrm{~d} z\right],
$$

where $k_{0}$ is the propagation constant in free space. Validity of this simple WKB solution (1) will be examined in the final section. Notation $q$ is one of the solution of the Booker quartic equation which is written by

$$
\xi q^{4}+\eta q^{3}+\zeta q^{2}+\gamma q+\delta=0
$$

where

$$
\begin{aligned}
& \xi=U\left(U^{2}-Y^{2}\right)+X\left(n^{2} Y^{2}-U^{2}\right), \\
& \eta=2 n X Y^{2} S l, \\
& \gamma=-2 C^{2} n S X Y^{2} l, \\
& \zeta=-2 U(U-X)\left(C^{2} U-X\right)+2 Y^{2}\left(C^{2} U-X\right)+X Y^{2}\left(1-C^{2} n^{2}+S^{2} l^{2}\right), \\
& \delta=(U-X)\left(C^{2} U-X\right)^{2}-C^{2} Y^{2}\left(C^{2} U-X\right)-C^{2} X Y^{2} S^{2} l^{2} .
\end{aligned}
$$

The notations $U=1-j Z, X=\left(\omega_{\mathrm{p}} / \omega\right)^{2}, Y=\omega_{\mathrm{H}} / \omega, Z=v_{\mathrm{eff}} / \omega, S=\sin \theta_{\mathrm{i}}$ and $C=\cos \theta_{\mathrm{i}}$ are used here, where $\omega_{\mathrm{p}}$ is plasma angular frequency, $\omega_{\mathbf{H}}$ is gyro angular frequency, $\omega$ is the angular frequency of the radio wave, $(l, m, n)$ are the direction cosines of the earth magnetic field, $\theta_{\mathrm{i}}$ is the angle of incidence and $v_{\text {eff }}$ is the effective collision frequency which is different from the average collision frequency.

The electron energy distribution are not taken into account in the coefficients for the Booker quartic Eq. (2). In practice, however, the momentum transfer cross section for electrons in the neutral particles of the $D$ region are proportional to the electron velocity (Phelps and PACK, 1959; Benson, 1964). In the statistical treatment of the collision of electrons with neutral particles by taking electron velocity distribution into account, it is convenient to use the collision frequency $v_{\mathrm{m}}$ for the mono-energetic electrons with energy 


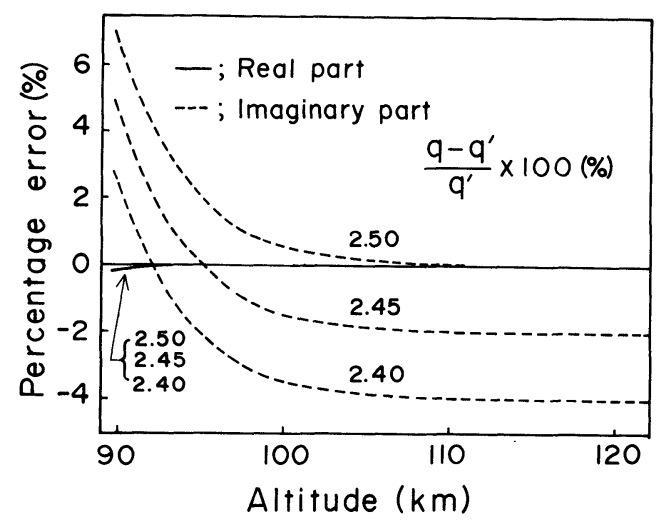

Fig. 4. Percentage errors of a standard Booker solution $q$ for a generalized Booker solution $q^{\prime}$ in the cases of $v_{\text {eff }}=2.4 v_{\mathrm{m}}, 2.45 v_{\mathrm{m}}$ and $2.5 v_{\mathrm{m}}$ when $f=40 \mathrm{kHz}, f_{\mathrm{H}}=1.2 \mathrm{MHz}$, the dip angle is $40^{\circ}$ and the angle of incidence is $82^{\circ}$. Solid and broken curves denote real and imaginary parts of the percentage errors, respectively.

$\kappa T$, where $\kappa$ is the Boltzman constant and $T$ is the electron temperature. The generalized Appleton-Hartree formula, which is the function of $v_{\mathrm{m}}$, has been led by using such a treatment (SEN and WYLLER, 1960; BUDDEN, 1965).

In order to apply the standard Booker equation, Eq. (2), in place of the generalized one with energy dependent collision frequency to our problem, we examined the relation between $v_{\text {eff }}$ and $v_{\mathrm{m}}$ by using Sen and Wyller theory for a whistler mode wave above the altitude $90 \mathrm{~km}$. The altitude distribution of the percentage deviation in the standardBooker solution from the generalized one is shown in Fig. 4 when $v_{\text {eff }}$ is $2.4 v_{\mathrm{m}}, 2.45 v_{\mathrm{m}}$ and $2.5 v_{\mathrm{m}}$. In this case, $v_{\mathrm{m}}$ profile is taken from Thrane and Piggott model and an electron density profile is assumed to be an exponential model: $N=10^{3} \mathrm{e}^{\alpha(z-90)} / \mathrm{cc}$, where $\alpha$ is $0.23 / \mathrm{km}$.

As shown in Fig. 4, the error of the $\operatorname{Im}(q)$ in the case of $v_{\text {eff }}=2.5 v_{\mathrm{m}}$ is about $5 \%$ at an altitude of $90 \mathrm{~km}$, but this error decreases abruptly as the altitude increases. Further, the errors in $\operatorname{Re}(q)$ are very small all over the region. On the other hand, the errors in $\operatorname{Im}(q)$ for the other cases are larger than those. Therefore, it is permissible in our problem that the collision term $v_{\text {eff }}$ in the standard Booker equation is replaced by $2.5 v_{\mathrm{m}}$, hence we use hereafter Eq. (2) to estimate the wave intensities.

For a whistler mode wave, $q$ in Eq. (1) must be appropriately selected in such a way that the root corresponding to the up going mode with right-handed circular polarization is selected out of four Booker's solutions. The complex variable $q$ is expressed as the sum of its real and imaginary parts, i.e., $q=q_{\mathrm{r}}-j q_{\mathrm{i}}$, where $q_{\mathrm{r}}$ and $q_{\mathrm{i}}$ are real and positive values. Since $q_{\mathrm{r}}$ is much greater than $q_{\mathrm{i}}$ in the upper $D$ and $E$ regions, the absolute value of the magnetic field in Eq. (1) is given by

$$
H=H_{1} q^{1 / 2} \exp \left[-k_{0} \int q_{\mathrm{i}} \mathrm{d} z\right],
$$

where $H_{1}$ is the intensity of the magnetic field at the altitude $z_{1}$. The above equation indicates that the amplitude of the magnetic field is proportional to the square root of a 
Booker's solution when the collision frequency is neglected.

To analyze the observed magnetic field intensities by using the Eq. (3) it is assumed that the ionosphere is represented as a large number of very thin homogeneous slabs of ionization. If we take the altitude of the base of the ionosphere as $z_{1}$, that of the $n$-th slab as $z_{n}$ and the slab thickness as $h$, then the ratio of the magnetic field in the $(i+1)$-th slab to that in the $i$-th slab is given by

$$
\frac{H\left(z_{i+1}\right)}{H\left(z_{i}\right)}=\left(\frac{q_{\mathrm{r}}\left(z_{i+1}\right)}{q_{\mathrm{r}}\left(z_{i}\right)}\right)^{1 / 2} \exp \left[-k_{0} q_{\mathrm{i}}\left(z_{i}\right) h\right] .
$$

The left term of the above equation is given by the observed magnetic field. Now suppose that the collision frequency $v_{\mathrm{m}}$ for mono-energetic electrons is proportional to the atmospheric pressure, $\mathrm{P}$, i.e., $v_{\mathrm{m}}=C \times 10^{5} \mathrm{P}$, where the value of $C$ is a collision proportionality factor, and $P$ is usually given from the CIRA model (1972) taking account of monthly variations. We consider how to get the value of $C$ under the condition that the electron density is not continuously observed in altitudes but there are observed data at independent altitudes.

\subsection{Iteration method}

Let $z_{i}$ and $z_{i+s}$ be the starting and the ending altitude of the iterative calculation, respectively, and the electron densities at these altitudes are assumed to be given.

By substituting the electron density observed at the altitude $z_{i}$ in Eq. (2), we first calculate a complex solution of the Booker quartic, $q\left(z_{i}\right)=q_{\mathrm{r}}\left(z_{i}\right)-j q_{\mathrm{i}}\left(z_{i}\right)$, based on adequate value of the collision proportionality factor. Since the left terms of $H\left(z_{i}\right)$ and $H\left(z_{i+1}\right)$ of Eq. (4) are given as the observed values, we can solve for the real part $q_{\mathrm{r}}\left(z_{i+1}\right)$ of the next slab by substituting these obtained $q_{\mathrm{r}}\left(z_{i}\right)$ and $q_{\mathrm{i}}\left(z_{i}\right)$ into the right term. To find the electron density in the next $(i+1)$-th slab from thus obtained $q_{\mathrm{r}}\left(z_{i+1}\right)$, we modify the previous Eq. (2) as the equation for $X$ which is defined as the square of the ratio of the plasma frequency to the radio wave frequency. The highest power of $X$ in the coefficients of Eq. (2) is $X^{3}$, so the equation is a cubic for $X$, which can be written:

$$
X^{3}+\alpha X^{2}+\beta X+\varepsilon=0,
$$

where

$$
\begin{aligned}
\alpha= & 2\left(q^{2}-C^{2}\right)-1, \\
\beta= & \left(1-n^{2} Y^{2}\right) q^{4}-2 \ln S Y^{2} q^{3}-\left[2\left(1+C^{2}\right)+Y^{2}\left(S^{2} l^{2}-C^{2} n^{2}-1\right)\right] q^{2} \\
& +2 C^{2} \ln S Y^{2} q+2 C^{2}-C^{2} Y^{2}+(l S C Y)^{2}+C^{4}, \\
\varepsilon= & -\left(1-Y^{2}\right) q^{4}-2 C\left(Y^{2}-1\right) q^{2}-C^{4}+C^{2} Y^{4} .
\end{aligned}
$$

In deriving the above cubic equation, we neglected the collision term because the imaginary part of $q$ is very small compared with the real part. The above equation has usually three real roots, and we can easily choose a solution corresponding to the electron density which is the closest to the $i$-th slab.

Similarly, we can calculate the electron density step by step to the altitude $z_{i+s}$. Then, 
a comparison is made of the electron density thus obtained and the observed one. If there is a difference between these two, the postulated collision proportionality factor is altered until the calculated electron density is in reasonable agreement with the observed electron density by the iterative procedure.

\section{Results and Discussion}

To get the collision frequency profile for mono-energetic electrons, we have used the atmospheric pressure obtained in August, at a latitude of $30^{\circ} \mathrm{N}$, for the CIRA model (1972) and then we have assumed that the each collision proportionality factor, $C$, is constant for two altitude ranges from $90 \mathrm{~km}$ to $106 \mathrm{~km}$ and from $106 \mathrm{~km}$ to $260 \mathrm{~km}$, respectively. We have calculated the electron density for altitudes from $90 \mathrm{~km}$ to $110 \mathrm{~km}$ and then we have obtained the collision proportionality factor by letting the calculated electron density agree with the observed one at the altitude of $106 \mathrm{~km}$ in the Table 1 . Figure 5 shows the calculated electron density profiles for various collision proportionality factors. As is seen in Fig. 5, the calculated maximum electron density agrees

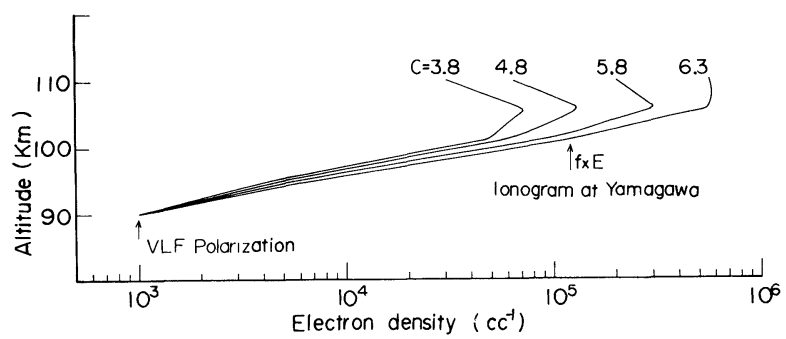

Fig. 5. Extrapolated electron density profile for various collision proportionality factors. The arrows denote the observed electron density.

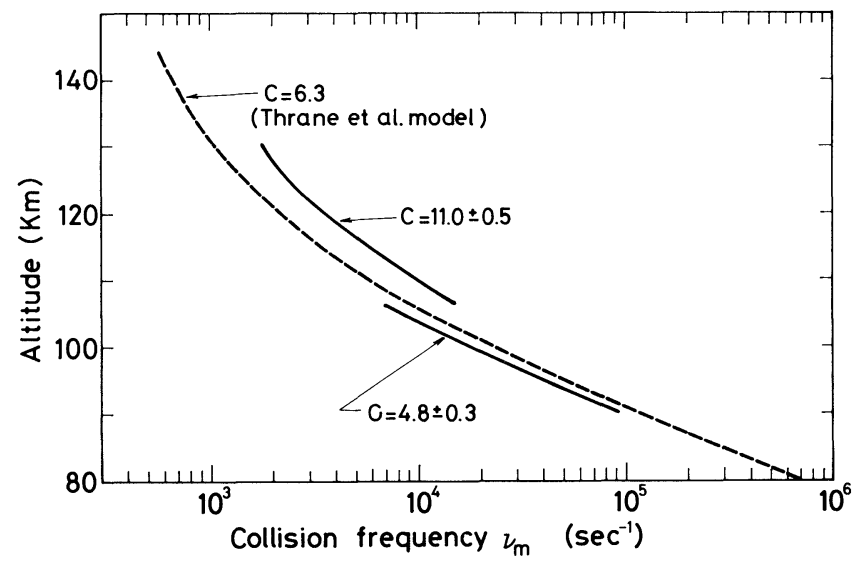

Fig. 6. Collision frequency profiles for mono-energetic electrons, $v_{\mathrm{m}}=C \times 10^{5} \mathrm{P}$, where $\mathrm{P}$ is an atmospheric pressure and taken from CIRA model (1972). Solid lines denote our results and the broken line shows Thrane and PiggotT's model (1966). 
with the ionogram data when the value of $C$ is 4.8 . Similarly, by making the iterative procedure from the altitude $106 \mathrm{~km}$ to $260 \mathrm{~km}$, we have finally got the value of $C$

$$
\begin{aligned}
& C=4.8 \pm 0.3, \quad 90 \mathrm{~km}<z<106 \mathrm{~km}, \\
& C=11.0 \pm 0.5, \quad 106 \mathrm{~km}<z<130 \mathrm{~km} .
\end{aligned}
$$

where $z$ is the altitude in $\mathrm{km}$.

The profiles of the collision frequency thus obtained are shown in Fig. 6. The collision frequency above an altitude of $130 \mathrm{~km}$ is not presented in Fig. 6, because above this altitude the absorption of $40 \mathrm{kHz}$ signal due to the collision is very small. The deviations from the mean values of $C$ in Eq. (6) correspond to the ranges of the accuracy of the observed electron densities in Table 1 which are assumed to contain measuring errors of $\pm 10 \%$. A comparison was made between our results and the value of $C(C=6.3)$ obtained from the laboratory measurement of cross section (THRANE and PIGGOTT, 1966) and is shown in the same figure. Our result is 15 per cent smaller than Thrane and Piggott's value for altitudes below $106 \mathrm{~km}$ and rather larger for altitudes higher than $106 \mathrm{~km}$. This may be due to the assumption that the atmospheric pressure consists only of the pressure of nitrogen molecule. The collision frequency above $106 \mathrm{~km}$ has a tendency to fit the reports (Thrane and Piggott, 1966; Mitra, 1970) in which they conclude that the experimental values of $v_{\mathrm{m}}$ at the $E$ region are inconsistent with the pressure variations of the standard atmosphere.

In terms of the definition of the collision frequency, the average collision frequency $v_{\mathrm{av}}$ is an important factor in plasma physics, yet it is difficult to arrive at the exact $v_{\mathrm{av}}$ in the ionosphere from radio wave experiment. If the momentum transfer cross section of the atmospheric gas is proportional to the velocity of the electron, it can be theoretically shown that $v_{\mathrm{av}}=3 / 2 v_{\mathrm{m}}$ (BUDDEN, 1965). On the other hand, in calculating the collisional damping of the radio wave in the lower ionosphere by the classical magneto-ionic theory, mono-energetic collision frequency profile of $v_{\mathrm{m}}$ has been often used because the relation

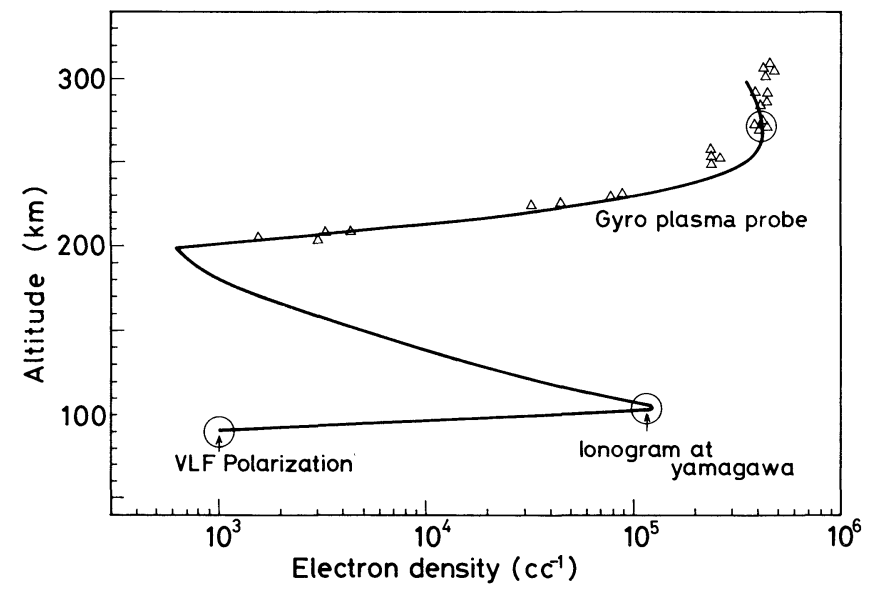

Fig. 7. The electron density profile extrapolated continuously by the iterative calculations. The arrows and triangles denote the observed electron densities. 


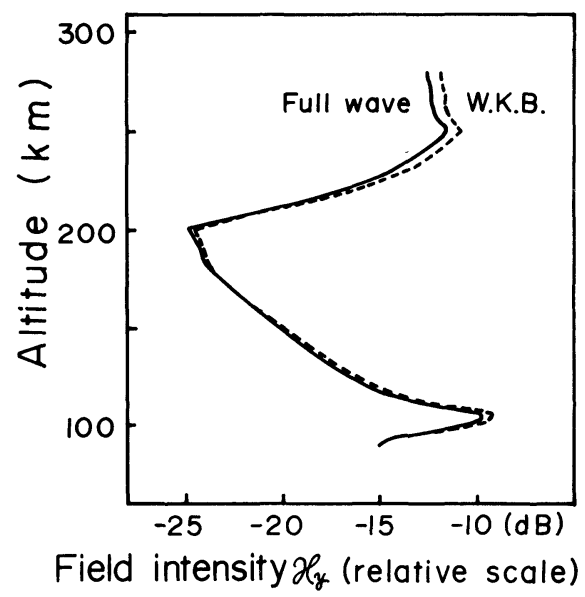

Fig. 8. Comparison between a full wave solution and a simple WKB solution Eq. (1) for a whistler mode wave above an altitude of $90 \mathrm{~km}$ with the ionospheric model deduced in this paper. Solid and broken curves show a full wave and a WKB solution, respectively.

between $v_{\text {eff }}$ and $v_{\mathrm{m}}$ has been made clear under certain conditions (SEN and WYLLER, 1960; DEEKS, 1966).

Figure 7 shows the electron density profile exptrapolated continuously by the process of iterative calculations. It is obvious that this profile agrees with the electron densities measured by gyro probe technique above the altitude of $200 \mathrm{~km}$.

As a final discussion, in order to check the validity of the WKB solution in Eq. (1) for a whistler mode above an altitude of $90 \mathrm{~km}$, we will present a result of the full wave technique which is capable of separating each characteristic mode from the total field (NAGANo et al., 1975).

Figure 8 shows the intensity variations of the whistler mode wave calculated by both methods for the ionospheric models which are shown in Figs. 6 and 7. The intensity of the field by the WKB solution is about $0.5 \mathrm{~dB}$ larger than that of full wave calculation at the altitude of $106 \mathrm{~km}$. But the difference in the field intensity between both methods does not exceed $1 \mathrm{~dB}$ all over the interesting altitudes, so that a simple WKB solution is proved to be a good approximation to the field intensity for the whistler mode waves.

\section{Summary}

A method of estimation of the collision frequency has been described, which requires the observed data for both the magnetic field intensity profile of a ground based signal and the ionospheric electron densities at several altitudes. Actually the collision frequency for mono-energetic electrons was estimated from the signal intensity profile of a $40 \mathrm{kHz}$ ground based signal observed by means of the K-9M-53 rocket. In analyzing the signal intensities, we have used a simple WKB solution.

In our method, the electron density need not be measured continuously. It is possible 
to use the ionogram data observed on the ground near the launching site in place of the electron density data observed by rocket. In this case, the instrument on board the rocket becomes very simple because only the measurement of the magnetic field intensity of a VLF signal is necessary.

We express our sincere thanks to the rocket launching staffs of the Institute of Space and Aeronautical Science, University of Tokyo. Thanks are also due to the Radio Research Laboratories and Japanese Defence Board for permitting special transmission of $40 \mathrm{kHz}$ standard signal. We wish to thank Professor $\mathrm{H}$. Oya of Tohoku University for providing us with the electron density data.

The computation was performed at the Data Processing Center, Kanazawa University.

\section{APPENDIX A}

A WKB solution for an upgoing whistler mode wave above an altitude of $90 \mathrm{~km}$

In a slowly varying medium, WKB solution for a general case associated with a characteristic mode corresponding to Booker root $q_{j}$ was given by BUDDEN (1961), equation (18-86).

$$
\begin{aligned}
\left(E_{x},-E_{y},\right. & \left.\mathscr{H}_{x}, \mathscr{H}_{y}\right) \\
& =\left(A_{j} F_{j}\right)^{1 / 2}\left(a_{3} q_{j}+a_{4}, A_{j}, q_{j} A_{j}, a_{5} q_{j}+a_{6}\right) \exp \left[-j k_{0} \int q_{j} \mathrm{~d} z+k_{0} \int \Gamma_{j j} \mathrm{~d} z\right],
\end{aligned}
$$

where $A_{j} F_{j}$ are some functions of $a_{1 \sim 6}$ and $b_{1 \sim 6}$, which are defined by BUDDEN (1961), Section 18-11.

We can simply modify Eq. (A.1) for an upgoing whistler mode wave above the upper $D$ region when the Earth magnetic field is not horizontal. In such region, it can be assumed that the ionospheric parameter $1-j Z$ is much less than $X$ and $Y$, and that $X \gg 1$. Then Eq. (A.1) become a simple form as follows

$$
\begin{aligned}
& \left(E_{x},-E_{y}, \mathscr{H}_{x}, \mathscr{H}_{y}\right) \\
& \quad=A_{0}\left(\rho \rho^{*}+1\right)^{1 / 2} q^{-1 / 2}(\rho, 1, q, \rho q) \exp \left[-j k_{0} \int q \mathrm{~d} z-j k_{0} \int\left(S m X^{\prime} / 4 q^{2} n^{2} Y\right) \mathrm{d} z\right]
\end{aligned}
$$

where $A_{0}$ is a constant, the superscripts "*" and "'" denote complex conjugate and the derivative of $X\left(\equiv \omega_{\mathrm{p}}{ }^{2} / \omega^{2}\right)$ with respect to altitude $z$, respectively, and $\rho$ is the ratio of $E_{x}$ to $E_{y}$. Direction cosines of the Earth magnetic field are $(l, m, n)$ and $S=\sin \theta_{\mathrm{i}}$. The second term of the exponent in Eq. (A.2) is neglected in a slowly varing medium.

In our $40 \mathrm{kHz}$ propagation experiment, the conditions for validity of Eq. (A.2), i.e., $Z<1, X>50$ and $Y=30$, are satisfied above an altitude of $90 \mathrm{~km}$, and as seen in Fig. 2, the right-handed circularly polarized wave $R$ is only detected above the altitude $90 \mathrm{~km}$, so that $\rho$ is almost pure imaginary and its magnitude is unity. Therefore a WKB solution for the whistler mode wave can be simplified as

$$
\mathscr{H}_{y} \simeq j A_{0}(2 q)^{1 / 2} \exp \left[-j k_{0} \int q \mathrm{~d} z\right] \text {. }
$$

Equation (A.3) agrees with a WKB solution for vertical incidence when the Earth magnetic field is parallel to the wave vector. 


\section{REFERENCES}

BENSON, R. F., Electron collision frequency in the ionospheric D region, Radio Sci., 68, 10, 1123-1126, 1964. Beynon, W. J. G. and E. R. Williams, Rocket measurements of $D$ region electron density profiles, J. Atmos. Terr. Phys., 38, 1318-1325, 1976.

BRemer, J. and W. Singer, Diurnal, seasonal and solar-cycle variations of electron densities in the ionospheric D- and E-regions, J. Atmos. Terr. Phys., 39, 25-34, 1977.

Budden, K. G., Radio Wave in the Ionosphere, $402 \mathrm{pp}$, Cambridge University Press, 1961.

Budden, K. G., Effect of electron collisions on the formulas of magneto-ionic theory, Radio Sci., 69D, 2, 191-210, 1965.

CIRA, COSPER international reference atmosphere, 196 pp., Berlin, Acadimie-verlag, 1972.

DeEKs, D. G., Generalised full wave theory for energy-dependent collision frequencies, J. Atmos. Terr. Phys., 28, 839-846, 1966.

Mechtly, E. A., Accuracy of rocket measurements of lower ionosphere electron density, Radio Sci., 9, 3, 373-378, 1974.

Mitra, A. R., HF and VHF absorption techniques in radio probing of the ionosphere, J. Atmos. Terr. Phys., 32, 623-646, 1970.

Nagano, I., M. MAmbo, and G. Hutatsuishi, Numerical calculation of electromagnetic waves in an anisotropic multilayered medium, Radio Sci., 10, 611-617, 1975.

NaGAno, I., M. Mambo, and I. Kimura, The electron density profile in the lower ionosphere derived from a rocket measurement of VLF propagation modes, Trans. IECE Japan, E59, 6-7, 1976.

NaGano, I., M. Mambo, and I. Kimura, Rocket measurements of lower ionosphere electron density by propagation characteristics of VLF waves, Planet. Space Sci., 26, 219-227, 1978.

PhelPS, A. V. and J. L. PACK, Electron collision frequencies in nitrogen and in the lower ionosphere, Phys. Rev. Lett., 3, 340-342, 1959.

SEN, H. K. and A. WYLLER, On the generalization of the Appleton-Hartree magnetoionic formular, J. Geophys. Res., 65, 3931-3950, 1960.

Thomas, L. and M. D. Harrison, The electron density distributions in the $D$-region during the night and presunrise period, J. Atmos. Terr. Phys., 32, 1-14, 1970.

Thrane, E. V. and W. R. Piggott, The collision frequency in the $E$ and $D$ region of the ionosphere, J. Atmos. Terr. Phys., 28, 721-737, 1966. 